\title{
A importância do pré-natal na saúde básica: uma revisão bibliográfica
}

\author{
The importance of prenatal in basic health: a bibliographic review \\ La importância de la prenatal em la salud básica: uma revisión bibliográfica
}

Recebido: 28/10/2021 | Revisado: 06/11/2021 | Aceito: 10/11/2021 | Publicado: 14/11/2021

\author{
Lucas de Moraes Escorcio Brito \\ ORCID: https://orcid.org/0000-0003-0995-8816 \\ Instituto de Educação Superior do Vale do Parnaíba, Brasil \\ E-mail: lucasmoraesescorcio@ outlook.com \\ Karla Karine Castelo Branco Mesquita \\ ORCID: https://orcid.org/0000-0003-2674-4967 \\ Instituto de Educação Superior do Vale do Parnaíba, Brasil \\ E-mail: karlinha2648@hotmail.com \\ Joérique Saraiva Melo \\ ORCID: https://orcid.org/0000-0002-4805-0803 \\ Instituto de Educação Superior do Vale do Parnaíba, Brasil \\ E-mail: joeriquemelo@gmail.com \\ Thainá Pinto dos Santos \\ ORCID: https://orcid.org/0000-0002-8460-4471 \\ Instituto de Educação Superior do Vale do Parnaíba, Brasil \\ E-mail: thainapsantos22@gmail.com
}

\begin{abstract}
Resumo
O início do pré-natal se deu no século XX, onde a saúde da mulher e do feto era fator preocupante, pois até então as taxas de mortalidade materna e infantil eram altas; todavia a finalidade do pré-natal era em reduzir estas taxas. Este presente estudo objetivou-se analisar o acompanhamento do pré-natal e seus desafios nas Unidades Básicas de Saúde, bem como analisar quais as ações preconizadas para a assistência à gestante, investigar as políticas de incentivo à assistência no pré-natal. Trata-se de uma revisão literária, no qual para o embasamento teórico foram selecionados artigos e textos científicos sobre os descritores relacionados ao assunto (Atenção Primária; Pré-Natal; Saúde da Mulher). Assim, mesmo com determinados riscos, são considerados momentos especiais na vida da mulher, a gravidez, o parto e o puerpério que são eventos fisiológicos que se desenvolvem em um contexto cultural e social. Nesse contexto, para que a assistência pré-natal seja considerada adequada e com um tratamento e acompanhamento que visa ser de forma holística, é necessário que ela seja precoce e assídua, além de contar com profissionais especializados, que realizem os tratamentos, internações e exames que se fizerem necessários. O Brasil é um país em desenvolvimento e mesmo o acesso não sendo de forma linear, é importante que os profissionais auxiliem a grávida da melhor forma. Ademais, o pré-natal tende a aumentar as chances de uma gravidez e parto mais tranquilos, com os riscos minimizados, sendo possível o tratamento precoce evitando sequelas e promovendo um parto mais seguro. Palavras-chave: Atenção primária; Pré-natal; Saúde da mulher.
\end{abstract}

\begin{abstract}
The beginning of prenatal care took place in the 20th century, when the health of women and fetuses was a worrying factor, since until then maternal and infant mortality rates were high; however, the purpose of pré-christmas care was to reduce these rates. This present study aimed to analyze the monitoring of pré-christmas care and its challenges in Basic Health Units, as well as to analyze which actions are recommended for the care of pregnant women, and to investigate the incentive policies for pré-christmas. This is a literary review, in which, for the theoretical basis, articles and scientific texts on the descriptors related to the subject were selected (Primary Care; Prenatal; Women's Health). Thus, even with certain risks, special moments in a woman's life are considered, pregnancy, childbirth and the puerperium, which are physiological events that develop in a cultural and social context. In this context, for préchristmas to be considered adequate and with treatment and monitoring that aims to be holistic, it is necessary that it beearly and diligent, in addition to having specialized professionals Who perform treatments, hospitalizations and examinations if necessary. Brazil is a developing country and even though access is not linear, it is important that professionals help pregnant women in the best way possible. Furthermore, pré-christmas tends to in crease the chances of a more relaxed pregnancy and childbirth, with minimized risks, with early treatment being possible, avoiding sequelae and promoting a safer childbirth.
\end{abstract}

Keywords: Primary care; Prenatal; Woman's health. 


\begin{abstract}
Resumen
El inicio de la atención prenatal se produjo em el siglo XX, cuando la salud de las mujeres y los fetos era um factor preocupante, ya que hasta esse momento las tasas de mortalidad materna e infantil eran elevadas; sin embargo, el propósito de la atención prenatal era reducir estas tasas. El presente estúdio tuvo como objetivo analizar el seguimiento de la atención prenatal y sus desafios em las Unidades Básicas de Salud, así como analizar qué acciones se recomiendan para la atención de la gestante e investigar las políticas de incentivos para La atención prenatal. Se trata de una revisión literaria, en la que, para la base teórica, se seleccionaron artículos y textos científicos sobre los descriptores relacionados com el tema (Atención Primaria; Prenatal; Salud de la Mujer). Así, incluso com ciertos riesgos, se consideran momentos especiales em la vida de la mujer, el embarazo, el parto y el puerperio, que son eventos fisiológicos que se desarrollan em un contexto cultural y social. En este contexto, para que la atención prenatal se considere adecuada y com um tratamiento y seguimiento que pretenda ser holístico, es necesario que sea precoz y diligente, además de contar com profesionales especializados que realicen tratamientos, hospitalizaciones y reconocimientos si es necesario. Brasil es un país em desarrollo y aunque el acceso no es lineal, es importante que los profesionales ayuden a las mujeres embarazadas de La mejor manera posible. Además, la atención prenatal tiende a aumentar lãs posibilidades de um embarazo y parto más relajado, com riesgos minimizados, siendo posible um tratamiento temprano, evitando secuelas y promoviendo un parto más seguro.
\end{abstract}

Palabras clave: Atención primária; Prenatal; La salud de la mujer.

\title{
1. Introdução
}

A assistência ao pré-natal, em consonância com o Ministério da Saúde, é o primeiro passo para um parto e nascimento saudável, ou seja, faz-se a manutenção e a promoção do bem-estar físico e emocional ao longo do processo da gestação, parto e nascimento, trazendo informação e orientação sobre a evolução da gestação e do trabalho de parto à parturiente. Assim, com o acompanhamento seguido de forma correta e ininterrupta, a gestante terá uma maior possibilidade de ter uma gestação mais saudável e tranquila. Ademais, um dos principais objetivos do pré-natal é acolher a mulher desde o início de sua gravidez, além de dar assistência em todas as suas necessidades. Vale ressaltar que cada gestante possui diferentes formas de lidar com esse período vivenciado (Brasil, 2000).

Cabe destacar também que a mulher durante o período gestacional tem o direito de possuir uma assistência técnica de qualidade, a partir do início da gravidez. Por isso é um dever do município se dispor de serviços de saúde que garantam a assistência pré-natal, parto, puerpério e neonatal, e que sejam prestados de forma devidamente organizados (Brasil, 2013).

No entanto, muitas gestantes que vivem nas ruas por exemplo, não possuem um pré- natal adequado e que a maioria delas estão sujeitas a riscos por conta da falta de orientação, falta de boas condições de higiene, uso exacerbado de drogas e álcool, o que pode consequentemente provocar alterações fetais e neonatais, doenças sexualmente transmissíveis e uma alimentação indevida, dificultando dessa forma um pré- natal adequado, sem riscos para mãe e para o bebê (Cardoso, 2019).

A gravidez é uma experiência única na vida de uma mulher e de sua família, e durante toda a gestação ocorrerá mudanças fisiológicas, envolvendo todos os sistemas orgânicos, gerando expectativas, emoções, ansiedade, medos e descobertas, exigindo que todas as modificações que ocorram no corpo tenham uma compreensão profunda. Nesse período, portanto, é necessário oferecer atenção adequada à saúde da paciente (Balica \& Aguiar; 2019).

O preparo pro momento do parto é algo essencial e que deve ser trabalhado desde a primeira consulta de pré-natal, para que a mulher conduza o período de gestação com mais autonomia e que possa encarar esse momento com mais calma e tranquilidade, o que ajuda no desenvolvimento do trabalho de parto (de Abreu et al., 2021).

A Estratégia Saúde da Família (ESF) é a porta de entrada dos serviços de saúde, por meio da qual as pessoas devem obter serviços de saúde simples ou complexos para garantir a continuidade do cuidado, monitorar e avaliar a saúde materna e perinatal. Nesse contexto, a saúde da família é entendida como uma estratégia de adequação do modelo assistencial que visa desenvolver ações individuais e coletivas de vigilância e promoção da saúde incluindo a assistência pré-natal (Brasil, 2016).

Conforme o manual do Ministério da Saúde, o pré-natal deve ser iniciado no primeiro trimestre da gestação e as consultas devem ser agendadas para garantir o acompanhamento necessário para uma assistência efetiva. As consultas devem 
ser realizadas pelo menos uma vez no primeiro trimestre, duas vezes no segundo trimestre e três vezes no terceiro trimestre, totalizando pelo menos seis consultas durante a gestação (Silva \& Pegoraro, 2018).

Se não houver consulta no início da gestação e não for possível uma avaliação sequencial do binômio materno-fetal, além de não ser possível detectar algumas doenças, o monitoramento do desenvolvimento fetal também acaba sendo prejudicado, podendo gerar grandes prejuízos (como o aparecimento de patologias a exemplo da diabetes gestacional e préeclâmpsia na gestante) tornando-se grandes empecilhos para um parto de sucesso. Por isso um pré-natal bem realizado na atenção básica não reduz apenas complicações durante a gestação, mas também facilita a atuação de uma equipe multiprofissional atuante. Dessa forma, diminui-se a prevalência de riscos iminentes na hora do parto, bem como possíveis infecções. Assim, visa-se uma maior qualidade na hora do parto para a gestante, o feto e para equipe multiprofissional poder realizar esta tarefa com menores riscos e complicações (Rodrigues \& Nascimento \& Araújo, 2011).

Seguindo esse pressuposto a pesquisa tem como objetivo geral analisar o acompanhamento do pré-natal e seus desafios nas Unidades Básicas de Saúde; e como específicos, analisar quais as ações preconizadas para a assistência à gestante no pré-natal; investigar as políticas de incentivo à assistência no pré-natal. Desenvolver através da literatura pertinente, dados sobre o pré-natal.

\section{Metodologia}

Trata-se de uma pesquisa bibliográfica. Sendo ela, entendida como uma revisão da principal literatura teórica que norteia o trabalho científico. Essa revisão é chamada de levantamento bibliográfico ou revisão bibliográfica e podem ser realizados em livros, periódicos, artigos de jornais, sites da Internet e outras fontes.

Conforme esclarece Boccato (2006, p. 266):

A pesquisa bibliográfica procura resolver problemas (hipóteses) através da publicação de referências teóricas, analisando e discutindo várias contribuições científicas. Este tipo de pesquisa fornecerá subsídios para o conhecimento estudado, como e sob que enfoque e / ou ponto de vista são tratados os temas apresentados na literatura científica. Para tanto, é imprescindível que os pesquisadores planejem sistematicamente o processo de pesquisa, abrangendo desde a definição do tema, passando pela construção lógica do trabalho até a determinação de sua disseminação e forma de divulgação.

Por ser a pesquisa bibliográfica um trabalho abrangente de busca de conhecimento, ela é à base de toda a pesquisa, portanto. Em primeiro lugar, o momento do livro deve ser elevado à máxima importância; mas seu propósito é também tornálo um objeto para facilitar o trabalho de quem pode ter dificuldade em localizar, identificar e gerenciar os bancos de dados existentes dos usuários.

A abordagem metodológica escolhida é a pesquisa qualitativa, pois essa abordagem proporciona que o investigador ajuste suas pesquisas na compreensão da área verdadeira, preocupando-se de acordo com o caráter hermenêutico na função de observar sobre o conhecimento vivido das pessoas e sociedade. Com isso, Ludke, retrata que:

A abordagem qualitativa parte do fundamento de que há uma relação dinâmica entre o mundo real e o sujeito, uma interdependência viva entre o sujeito e o objeto, um vínculo indissociável entre o mundo objeto e a subjetividade do sujeito (Ludke \& Marli, 1996).

Ou seja, a pesquisa qualitativa está mais associada na relação de informações sobre os incentivos de um conjunto, em entender e compreender práticos comportamentos, a pensamento e as perspectivas das pessoas de um cidadão. A pesquisa qualitativa é excelente para investigar informações já calculadas ou em que se deseja estabelecer um conhecimento de prática para só posteriormente quantificá-los. 
A metodologia é o caminho que o pesquisador percorre para atingir o seu objetivo proposto (Lakatos \& Marconi, 2003). Neste trabalho, optamos por uma pesquisa de natureza básica, abordagem qualitativa, uma vez que ela dá maior flexibilidade de busca do material a ser analisado. Este foi coletado na Biblioteca Virtual em Saúde (BVS), nas bases dedados da literatura Latino-Americana e do Caribe em Ciências da Saúde (LILACS) e no Scientific Eletronic Library Online (SciELO).

Trata-se de uma revisão litererária, no qual para o embasamento teórico foram selecionados artigos e textos científicos sobre os descritores relacionados ao assunto. Optou-se por utilizar como material, artigos científicos produzidos entre 2010 e 2021 em língua portuguesa e livros científicos, por considerar mais acessíveis este tipo de publicação para os profissionais de saúde, além dos manuais do Ministério da Saúde desde 2000 até 2013. Para a busca do embasamento teórico temos os seguintes descritores: Atenção Primária; Pré-Natal; Saúde da Mulher.

\section{Resultados e Discussão}

O início do pré-natal se deu no século XX, onde a saúde da mulher e do feto era fator preocupante, pois até então as taxas de mortalidade materna e infantil eram altas; todavia a finalidade do pré-natal era em reduzir estas taxas (Galleta, 2000).

Assim, mesmo com determinados riscos, são considerados momentos especiais na vida da mulher, a gravidez, o parto e o puerpério que são eventos fisiológicos que se desenvolvem em um contexto cultural e social que determina e influencia a evolução da gravidez bem como a sua assistência (Dourado, 2005).

Nesse contexto, segundo Barbosa (2007), a gravidez provoca inúmeras transformações no corpo, psicológico e estado emocional da mulher, além de modificar também todo o contexto socioeconômico e cultural no qual se encontra. Barbosa cita também que cada mulher vive esse processo gestacional de maneira diferente e a combinação destes fatores torna este momento especial e único. Dessa forma, a assistência deve ser holística (ou seja, de forma abrangente em todos os seus aspectos), individualizada e todos esses fatores devem ser levado em conta pelos profissionais que mantém o acompanhamento.

O Manual Técnico do Pré-Natal e Puerpério do Ministério da Saúde preconiza:

O Ministério da Saúde recomenda iniciar acompanhamento da gestante no primeiro trimestre de gravidez e a realizar pelo menos seis consultas (sendo, no mínimo, duas realizadas por médico). Os principais procedimentos recomendados para as consultas são: exame físico (peso e estado nutricional da gestante; estatura; pulso e temperatura; pressão arterial; inspeção de pele, mucosas e tireóides; ausculta cárdio-pulmonar; exame de membros inferiores), exame ginecológico (exame de mamas, altura uterina, batimentos cárdio-fetais, palpação de gânglios e genitália, exame especular); exames laboratoriais de rotina (tipagem sanguínea, VDRL, urina e hemoglobina). Todas as gestantes devem receber segundo estas normas, suplementação de ferro (independentemente do nível de hemoglobina) e orientação quanto ao aleitamento materno, entre outros procedimentos. Serão feitos exames de secreção vaginal, "preventivo de câncer de colo de útero" e vacina antitetânica apenas se houver indicação (Brasil, 2005, p. 23).

Dessa forma, é importante seguir o Manual Técnico do Pré-Natal e Puerpério do Ministério da Saúde, a fim de minimizar os possíveis riscos gravídicos, além de acompanhar o processo gestacional visando um parto saudável, com chances reduzidas de complicações.

Grande parte dos serviços de saúde tem passado por mudanças, na estruturação e organização, objetivando construir um modelo de atenção à saúde mais equânime, justa, democrática, participativa e que tenha como prerrogativas básicas os princípios da humanização da assistência, promoção e prevenção em saúde (Barbosa \& Gomes \& Dias, 2011). Na assistência da atenção primária (AP) a realização das diretrizes e princípios do SUS, tem desenvolvimento em um processo contínuo na avaliação do serviço de pré-natal, tendo assim um atendimento integral à usuária, implicando uma prática de qualidade melhor 
e desenvolvendo a capacidade de análise crítica de contextos. (Pinho \& Siqueira \& Oliveira, 2006).

O Ministério da Saúde (MS) estabeleceu o Programa de Humanização no Pré- Natal e no Nascimento, através da Portaria n. ${ }^{\circ}$ 569/ GM, de 1 de junho de 2000. Neste encontra-se inserido os princípios e diretrizes para a construção desse programa, que ditam os direitos da gestante como: escolha da maternidade onde ser atendida no parto, acompanhamento prénatal, atendimento humanizado no parto e puerpério além da adequada assistência neonatal ao recém-nascido. Nessa portaria, o MS ainda responsabiliza as autoridades sanitárias no âmbito municipal, estadual e federal pela garantia destes direitos e tendo como o maior objetivo oferecer um atendimento de qualidade e digno no decorrer da gestação, parto e puerpério (Brasil, 2000).

Os principais objetivos do Programa de Humanização do Pré-Natal e Nascimento são: aumentarem o acesso ao prénatal, listar critérios para melhorar as consultas, reduzir as taxas de morbimortalidade materna e perinatal, e gerar vínculo entre a assistência ambulatorial e o parto. O programa ressalta ainda quais são os mínimos procedimentos a serem ofertados para mulheres durante o ciclo gravídico-puerperal e proporciona como estratégias para a melhoria da qualidade da atenção respeito aos direitos reprodutivo e a humanização do cuidado prestado (Serruya., Cecatti \& Lago, 2004).

O acompanhamento pré-natal é de suma importância pois permite aumentar a garantia de um desenvolvimento saudável para a criança e certamente uma diminuição dos riscos para as gestantes, além de poder detectar patologias que acometiam a paciente anteriormente como também as desenvolvidas durante a gravidez, porém, com um quadro evolutivo silencioso, são exemplos, diabetes, anemias, hipertensão arterial, sífilis, doenças do coração, dentre outras. Assim, o diagnóstico precoce permite a implementação de um tratamento eficaz no intuito de evitar um maior prejuízo a mulher e ao concepto, não só durante a gestação, mas por toda sua vida (Neumann et al., 2003). É ressaltado ainda que a troca de informações relacionadas às diferentes experiências entre as gestantes e os profissionais de saúde é visto como a melhor forma de promover a compreensão do processo de gestação (Cardoso, et al., 2007).

Além do diagnóstico precoce, hoje é possível, em algumas situações, o tratamento intra-úterino, que possibilita ao recém-nascido uma avaliação de aspectos relacionados à placenta e uma vida normal, permitindo a introdução de um tratamento adequado (Viellas, Domingues,2014). Outro benefício do pré-natal é a possibilidade de identificar precocemente eclâmpsia (a principal patologia responsável pela mortalidade durante o período da gravidez no Brasil). Esta patologia se caracteriza pela elevação da pressão arterial da gestante, ocasionando um comprometimento da função renal e cerebral, chegando até mesmo a quadros de convulsões e coma (Caldeira et al., 2010).

Além das normas, o Ministério da Saúde cita também os principais objetivos das consultas pré-natais: a disseminação de informações educativas a respeito do parto e do cuidado da criança, trazendo informações sobre os hábitos de higiene e vida, a preparação psíquica e física da mulher para a maternidade, e ainda de manutenção do estado nutricional adequado (Brasil, 2006). Além disso, através das consultas pré-natais obtêm-se ainda a orientação sobre o uso de medicações que possam afetar tanto o feto, como à mulher e a evolução do parto, e também o tratamento de manifestações físicas peculiares da gravidez, como os vômitos, as náuseas, as azias, as cãibras, as queixas respiratórias, de dores urinárias, lombares, mamarias, dentre outras.

Outro programa que possui ações para serem realizadas durante esse período é o Sistema de Acompanhamento do Programa de Humanização no Pré-Natal e Nascimento (SisPreNatal) que tem como objetivos: desenvolvimento de Ações de Promoção, Prevenção e Assistência à Saúde dos Recém Nascidos e Gestantes, para que possam reduzir as taxas de mortalidade materna, bem como neonatal e perinatal, aprimorando o pré-natal nos âmbitos do acesso, cobertura e qualidade deste pré-natal, se estendendo até a assistência ao parto e puerpério, oferecendo as iniciativas aos Municípios, Estados e Ministério da Saúde, através de informações fundamentais ao planejamento, acompanhamento e avaliação de todas ações realizadas pelo Programa de Humanização no Pré-Natal e Nascimento (Cruz, 2019).

Segundo Rocha et al. (2011), em estudos realizados, mostraram que o pré-natal realizado adequadamente foi de 
apenas 35\% das usuárias da ESF (Estratégia Saúde da Família), mostrando a importância de intervenções para a adesão precoce das mulheres ao pré-natal. $\mathrm{O}$ tardio acompanhamento do pré-natal evidenciado afeta diretamente o acompanhamento e monitoramento da gestação. "O início precoce do pré-natal permite o acesso a métodos diagnósticos e terapêuticos para a prevenção das possíveis complicações gestacionais”. Além disso, permite a monitoração do crescimento fetal e fundamenta a tomada de decisão clínica relacionada ao desfecho da gestação.

Já em outro estudo realizado por Calderon, Cecatti, (2006) mostrou que de 50.791 mulheres incluídas na avaliação do PMAQ-AB (Programa de Melhoria do Acesso e da Qualidade) em todo o território nacional, 45.560 (89,8\%) já haviam engravidado e 8.777 (19,3\%) tinham filhos com menos de dois anos de idade; destas, 8.667 (98,9\%) realizaram o pré-natal na última gravidez e $6.125(70,7 \%)$ o fizeram em 4.924 UBS onde estavam sendo entrevistadas. Dessas mulheres, 43,6\% encontravam-se na faixa etária de 25 a 34 anos e $20 \%$ na de 16 a 20 anos.

A proporção de mulheres que recebeu todas as orientações foi de $60,3 \%$, sendo as orientações sobre amamentação exclusiva a mais oferecida, e sobre a importância do pré-câncer a menos oferecida. Assim nesse referido estudo, 69,2\% das entrevistadas afirmaram ter realizado todos os exames complementares durante o pré-natal, sendo a glicemia e o VDRL os exames com menor prevalência, e o comum de urina, anti-HIV e ultrassonografia registraram maior prevalência.

No Brasil, o exame anti-HIV deve ser realizado pelas gestantes, sendo solicitado na primeira consulta do pré-natal. Em caso de diagnóstico confirmado e positivo, as medidas específicas devem ser tomadas o mais rápido possível pelo profissional de saúde para que não se contaminem o parceiro e o bebê, além de iniciar o pré-natal de alto risco com maiores cuidados incluindo o tratamento contra a doença (Goncalves \& Piccinini, 2007).

Segundo Barbosa (2007) uma assistência pré-natal com qualidade não precisa necessariamente de procedimentos de alta tecnologia e complexos, afinal, uma das chaves da qualidade desta assistência está, sobretudo em um relacionamento de confiança entre os profissionais, a gestante e sua família.

Até 1983 as políticas públicas de saúde eram voltadas para a atenção materno-infantil, com a implantação do Programa de Assistência Integral à Saúde da Mulher (PAISM) houve uma diferenciada e nova abordagem da saúde da mulher onde a "atenção integral à saúde das mulheres" deveria contemplar problemas presentes desde a adolescência até a terceira idade; aspectos clínico-ginecológico e educativo, controle pré-natal, parto e puerpério; à assistência para concepção e contracepção e doenças transmitidas sexualmente, câncer cérvico-uterino e mamário. Foi a primeira vez que um programa se voltou para a regulação da fecundidade.

O Programa de Humanização do Pré-natal e Nascimento (PHPN) tem como princípio de embasamento a humanização da assistência obstétrica e neonatal para o adequado acompanhamento do parto e puerpério, e, que a partir de um atendimento holístico, mais humanizado e participativo o profissional deve, além daquilo que se vê e apalpa, ouvir e levar em consideração as dúvidas, dificuldades e ansiedades da gestante (Duarte \& Andrade, 2008).

Assim, tendo em base o que os autores Caldeira et al. (2010), Gonçalves et al. (2008) e Neumann et al. (2003) dentre outros afirmam, para que a assistência pré-natal seja considerada adequada e com um tratamento e acompanhamento que visa ser de forma holística, é necessário que ela seja precoce e assídua, além de contar com profissionais especializados e engajados, que realizem os tratamentos, internações e exames que se fizerem necessários. Além disto, uma área física adequada com equipamentos e instrumental, instrumentos de registro e estatística, medicamentos básicos e uma avaliação periódica e continua das ações da assistência pré-natal são fatores importantes para a qualidade da assistência prestada, visando um atendimento que chegue em todas as esferas de acompanhamento de gestantes de forma que vise a equidade e de forma satisfatória. 


\section{Conclusão}

Através do presente estudo pode-se enfatizar a importância de um bom pré-natal desde o início da gravidez, primeiro trimestre, evitando-se doenças ou tratando doenças pré-existentes minimizando os riscos iminentes. O engajamento da grávida aliado a sua vontade de conhecer os benefícios de um bom pré-natal favorece a sua adesão a realização de um bom acompanhamento. Assim, a participação de toda equipe de saúde médica e multiprofissionais engajados nessa causa contribui para um bom auxílio durante esta trajetória.

Dessa forma, o pré-natal evita maiores agravamentos e posteriores riscos tanto para a gravídica quanto para o feto, sendo este acompanhamento regido por políticas públicas. O Brasil é um país em desenvolvimento e mesmo o acesso não sendo de forma linear, é importante que os profissionais auxiliem a grávida da melhor forma, a fim de minimizar riscos e aumentar a adesão das consultas.

Geralmente a Estratégia de Saúde da Família é a porta de entrada para as mulheres e uma atenção adequada à mulher é indispensável para garantir que ela exerça a maternidade com segurança e bem-estar. A equipe de saúde deve acolher a mulher e sua família. Para tal se faz necessário a formação de um vínculo mais profundo com a gestante, que lhe transmita confiança e tranqüilidade.

Ademais, o pré-natal tende a aumentar as chances de uma gravidez e parto mais tranquilos, com os riscos minimizados, sendo possível o tratamento precoce evitando sequelas e promovendo um parto mais seguro.

\section{Referências}

Balica, L. O., \& Aguiar, R. S. (2019). Percepções paternas no acompanhamento do pré-natal. Revista de Atenção a Saúde, 17, 114-126.

Barbosa, M. A. (2007). Avaliação da assistência pré-natal de baixo risco no município de Francisco Morato. Universidade Guarulhos, Centro de PósGraduação, Pesquisa e Extensão. http://hdl.handle.net/123456789/246.

Brasil. (2002). Programa de humanização do pré-natal e nascimento. Ministério da Saúde. Secretaria de Políticas de Saúde. https://bvsms.saude.gov.br/bvs/publicacoes/parto.pdf

Brasil. (2013). Atenção ao pré-natal de baixo risco. Ministério da Saúde. Secretaria de atenção à saúde. https://bvsms.saude.gov.br/bvs/publicacoes/atencao_pre_natal_baixo_risco.pdf

Calderon, I. D. M. P., Cecatti, J. G., \& Vega, C. E. P. (2006). Intervenções benéficas no pré-natal para prevenção da mortalidade materna. Revista Brasileira de Ginecologia e Obstetrícia, 28, 310-315.

Caldeira, A. P., Oliveira. R. M. D., \& Rodrigues, O. A. (2010). Qualidade da assistência materno-infantil em diferentes modelos de Atenção Primária. Ciência \& Saúde Coletiva, 15, 3139-3147.

Cardoso, Â. M. R., Santos, S. M., \& Mendes, V. B. (2007). O pré-natal e a atenção à saúde da mulher na gestação: um processo educativo. Revista Diálogos Possíveis, 6(1), 141-159.

Cardoso, S. L., Souza, M. E., Oliveira, R. S., Souza, A. F., Lacerda, M. D., Oliveira, N. T., Castro, A. P., \& Medeiros, K. M. (2019). Ações De Promoção Para Saúde Da Gestante Com Ênfase No Pré-Natal. Revista Interfaces: Saúde, Humanas e Tecnologia. 7(1), $180-186$.

Cunha, M. D. A. (2008). Assistência pré-natal por profissionais de enfermagem no município de Rio Branco-AC: contribuição para o estudo da atenção qualificada no ciclo grávido-puerperal. Tese (Doutorado), Universidade de São Paulo.

Cruz, A. D. L. (2019). Importância da atenção pré-natal na prevenção de complicações na gestação: revisão integrativa. TCC (Graduação) Universidade Católica do Salvador Faculdade de Enfermagem. <http://ri.ucsal.br:8080/jspui/bitstream/prefix/940/1/TCCALESSANDRACRUZ.pdf>.

da Silva, A. C. D., \& Pegoraro, R. F. (2018). A Vivência do Acompanhamento Pré-Natal Segundo Mulheres Assistidas na Rede Pública de Saúde. Revista Psicologia e Saúde, 10, 95-107.

de Abreu, H. D. S. C., de Almeida, L. P., Mouta, R. J. O., Silva, S. C. D. S. B., Zveiter, M., Medina, E. T. \& dos Santos, L. L. (2021). Contribuição do prénatal no preparo da gestante para o trabalho de parto. Research, Society and Development, 10 (10), e405101017886-e405101017886.

Dourado, V. G., \& Pelloso, S. M. (2007). Gravidez de alto risco: O desejo e a programação de uma gestação. Acta Paulista de Enfermagem, $20,69-74$.

Duarte, S. J. H., \& Andrade, S. M. O. D. (2008). O significado do pré-natal para mulheres grávidas: uma experiência no município de Campo Grande - Brasil. Revista Saúde e Sociedade, 17, 132-139.

Goncalves, T. R., \& Piccinini, C. A. (2007). Aspectos psicológicos da gestação e da maternidade no contexto da infecção pelo HIV/Aids. Psicologia USP, 18, 113-142. 
Research, Society and Development, v. 10, n. 15, e51101522471, 2021

(CC BY 4.0) | ISSN 2525-3409 | DOI: http://dx.doi.org/10.33448/rsd-v10i15.22471

Neumann, N. A., Tanaka, O. Y., Victora, C. G., \& Cesar, J. A. (2003). Qualidade e equidade da atenção ao pré-natal e ao parto em Criciúma, Santa Catarina, Sul do Brasil. Revista Brasileira de Epidemiologia, 6, 307-318.

Pinho, I. C., Siqueira, J. C. B. A., \& Oliveira, P. L. M. (2006). As percepções do enfermeiro acerca da integralidade da assistência. Revista Eletrônica de Enfermagem, 8, 42-51.

Rodrigues, E. M., Nascimento, R. G. D., \& Araujo, A. (2011). Protocolo na assistência pré-natal: ações, facilidades e dificuldade dos enfermeiros da Estratégia de Saúde da Família. Revista da Escola de Enfermagem, 45, 1041-1047.

Serruya, S. J., Cecatti, J. G., \& Lago, T. D. G. D. (2004). O Programa de Humanização no Pré-natal e Nascimento do Ministério da Saúde no Brasil: resultados iniciais. Cadernos de Saúde Pública, 20, 1281-1289.

Silva, L. R. D., Christoffel, M. M., \& Souza, K. V. D. (2005). História, conquistas e perspectivas no cuidado à mulher e à criança. Revista Texto e Contexto Enfermagem, 14, 585-593.

Viellas, E. F., Domingues, R. M. S. M., Dias, M. A. B., Gama, S. G. N. D., Theme, M. M., Costa, J. V. D., \& Leal, M. D. C. (2014). Assistência pré-natal no Brasil. Caderno de Saúde pública, 30, 585-92. 